\title{
La escritura colaborativa en ambientes educativos presenciales, virtuales y con diferentes mediaciones de la tecnología digital $^{*}$
}

\author{
Pilar del Carmen Bonilla-Valencia** \\ Diana Patricia de Castro-Daza***
}

Recibido: 22-07-2020

Aceptado: 24-07-2020

\begin{abstract}
Citar como: Bonilla-Valencia, P. C. y de Castro-Daza, D. P. (2021). La escritura colaborativa en ambientes educativos presenciales, virtuales y con diferentes mediaciones de la tecnología digital.

Revista Interamericana de Investigación, Educación y Pedagogía, 14(2), 195-223. https://doi. org/10.15332/25005421.6061
\end{abstract}

\section{Resumen}

El artículo tiene como objetivo presentar una revisión bibliográfica sobre las tendencias, aportes y limitaciones de los estudios sobre escritura colaborativa en los últimos once años. En la metodología se definieron cuatro criterios de inclusión de los estudios: 1) el abordaje de la escritura colaborativa; 2) la fecha de publicación entre el año 2009 y 2020; 3) los artículos publicados en español, inglés y portugués; y 4) el tipo de artículo: investigación, revisión

\footnotetext{
* Artículo de revisión derivado de una parte del estado del arte realizado para el proyecto de tesis doctoral titulado Los procesos recursivos en la producción textual colaborativa entre niños mediada por tecnología digital del Doctorado en Psicología de la Universidad del Valle, Colombia.

** Psicóloga, magíster en Psicología con énfasis en Psicología Clínica y estudiante del Doctorado en Psicología de la Universidad del Valle. Miembro del grupo de investigación Lenguaje, Cognición y Educación adscrito al Centro de Investigaciones en Psicología, Cognición y Cultura de la Universidad del Valle.

Correo electrónico: pilar.bonilla@correounivalle.edu.co

ORCID: https://orcid.org/0000-0002-5436-1894

Google Scholar: https://scholar.google.com/citations?user=PWs $18 E g A A A A J \& h l=e s$

Currículo MinCiencias: https://scienti.minciencias.gov.co/cvlac/visualizador/generarCurriculoCv. do?cod_rh $=0001393258$

Iralis: https://www.iralis.org/app/ficha 17823

*** Doctora en Psicología con énfasis en Procesos de Desarrollo Humano y Salud por la Universidad de Brasilia, Brasil. Psicóloga y magíster en Psicología con énfasis en Psicología del desarrollo Cognitivo por la Universidad del Valle. Profesora del Instituto de Psicología y directora del grupo de investigación Lenguaje, Cognición y Educación adscrito al Centro de Investigaciones en Psicología, Cognición y Cultura de la Universidad del Valle.

Correo electrónico: diana.decastro@correounivalle.edu.co

ORCID: https://orcid.org/0000-0002-2213-5817

Google Scholar: https://scholar.google.com/citations?hl=es\&user=ZxC8n-IAAAAJ

Currículo MinCiencias: https://scienti.minciencias.gov.co/cvlac/visualizador/generarCurriculoCv. do?cod_rh $=0000301906$

Iralis: https://www.iralis.org/app/ficha 18000
} 
y teórico. Se realizó la búsqueda en las bases de datos: Scopus, ProQuest, Taylor \& Francis, Web of Science, JSTOR y Ebsco. Los resultados de la revisión evidencian que la escritura colaborativa no es un proceso que se facilita de manera automática por las herramientas y mediaciones digitales, sino que requiere de un ambiente educativo en el que los participantes se reconozcan como pares coautores del texto en una relación dialógica. El estudio de la escritura colaborativa conlleva a considerar aspectos relacionales entre los escritores, el propósito del escrito y la audiencia proyectada, así como la coherencia textual, lo que comprende una ampliación de la concepción de escritura. Entre las limitaciones se identificó que los estudios revisados no profundizan en la incidencia de la escritura colaborativa en la calidad textual ni en la argumentación en el proceso de producción. En las conclusiones se resalta que la escritura colaborativa plantea como reto potenciar, en los distintos niveles de formación, prácticas educativas que promuevan la construcción conjunta entre pares, la creatividad y el uso pedagógico y ético de la tecnología digital.

Palabras clave: escritura, tecnología educacional, comunicación interactiva, ambiente educacional, interacción social, relaciones entre pares.

\section{Collaborative Uriting in Face-to-Face and Virtual Educational Environments and With Different Mediations of Digital Technology}

\section{Abstract}

This article aims to present a bibliographic review on the trends, contributions, and limitations of studies on collaborative writing in the last eleven years. The methodology defined four criteria for the inclusion of the studies: 1) the collaborative writing approach; 2) a publication date between 2009 and 2020;3) articles published in Spanish, English, and Portuguese; and 4) the type of article: research, 
review, and theoretical. A search was conducted in the following databases: Scopus, ProQuest, Taylor \& Francis, Web of Science, JSTOR, and Ebsco. The results of the review show that collaborative writing is not a process that is facilitated automatically by digital tools and mediations but requires an educational environment in which the participants recognize themselves as co-author peers of the text in a dialogic relationship. The study of collaborative writing involves considering relational aspects between the writers, the purpose of the writing and the intended audience, as well as textual coherence, which implies an expansion of the conception of writing. Among the limitations, it was identified that the studies reviewed do not delve into the incidence of collaborative writing on textual quality or argumentation in the production process. The conclusions highlight that collaborative writing poses the challenge of encouraning, at different levels of training, educational practices that promote joint construction among peers, creativity, and the pedagogical and ethical use of digital technology.

Keywords: writing, educational technology, interactive communication, educational environment, social interaction, peer relationships.

\section{A escrita colaborativa em ambientes educativos presenciais, virtuais e com diferentes mediações da tecnologia digital}

\section{Resumo}

O artigo tem como objetivo apresentar uma revisão bibliográfica sobre as tendências, contribuições e limitações dos estudos sobre escrita colaborativa nos últimos onze anos. Na metodologia foram definidos quatro critérios de inclusão: 1) a abordagem da escrita colaborativa; 2) a data de publicação entre os anos de 2009 e 2020; 3) os artigos publicados em espanhol, inglês e português; e 4) o tipo de artigo: investigação, revisão e teórico. Foi realizada uma 
busca nas bases de dados: Scopus, ProQuest, Taylor \& Francis, Web of Science, JSTOR e Ebsco. Os resultados da revisão evidenciam que a escrita colaborativa não é um processo que é facilitado de forma automática pelas ferramentas e mediações digitais, mas que requer um ambiente educativo, em que os participantes se reconheçam como pares na coautoria do texto em uma relação dialógica. O estudo da escrita colaborativa implica considerar aspectos relacionais entre os escritores, o propósito do que é escrito e a audiência projetada, assim como a coerência textual; o que envolve uma ampliação da concepção de escrita. Entre as limitações, identifica-se que os estudos revisados não se aprofundam no impacto da escrita colaborativa na qualidade textual, nem na argumentação nesse processo de produção. Nas conclusões, ressalta-se que a escrita colaborativa representa um desafio para potencializar, nos distintos níveis de formação, práticas educativas que promovam a construção conjunta entre pares, a criatividade e o uso pedagógico e ético da tecnologia digital.

Palavras-chave: escrita, tecnologia educacional, comunicação interativa, ambiente educacional, interação social, relações entre pares.

\section{Introducción}

Este artículo tiene como propósito presentar una revisión bibliográfica de los estudios realizados en los últimos once años sobre la escritura colaborativa, en la que se identifiquen las tendencias, los aportes y las limitaciones en su abordaje. La escritura colaborativa es un tema de relevancia actual, que propicia avanzar en propuestas para la producción textual que consideren escenarios lúdicos y que fomenten la creatividad, la interacción entre pares y el uso de la tecnología digital en las prácticas educativas para promover la colaboración grupal. 
La escritura ha sido estudiada desde diferentes disciplinas y perspectivas. Donahue y Lillis (2014) identifican cuatro modelos explicativos desde los que ha sido abordada: modelo del texto que proviene de la lingüística, modelo del proceso didáctico de la educación, modelo (socio) cognitivo de diferentes perspectivas de la psicología y modelo de práctica social de la antropología. Dentro de los abordajes que clasifican las autoras en el modelo (socio) cognitivo, se sitúan aquellos derivados de la psicología cultural, que se sustenta en la teoría histórico-cultural de Vygotsky y en la teoría de la actividad de Leontiev. En estas perspectivas teóricas tienen un lugar preponderante el estudio de la interacción con los otros y la construcción de significados, aspectos constitutivos de los procesos colaborativos.

La escritura colaborativa es un proceso de construcción conjunta de sentido que tiene como referente teórico el aprendizaje colaborativo, el aprendizaje cooperativo, la perspectiva histórico-cultural de Vygotsky y la teoría de la actividad de Leontiev y Engeström (Vergara Novoa y Perdomo Cerquera, 2016). Los abordajes referidos hacen énfasis en el valor de la interacción social y la mediación en el aprendizaje, al igual que resaltan el andamiaje como un aspecto fundamental en la construcción social del conocimiento.

En este sentido, la escritura colaborativa es un proceso social e interactivo que requiere del establecimiento de trabajo en equipo y de un objetivo negociado durante la construcción del texto (Lowry, Curtis y Lowry, 2004). Los escritores trabajan juntos durante el proceso de la escritura, comparten la autoría y la responsabilidad del producto final (Storch, 2019). En esta vía, resulta pertinente resaltar la dialogicidad en el proceso de escritura colaborativa. Bajtín (1999) advierte que los sujetos se construyen en la relación dialógica con los otros; es decir, en una relación en la que emergen intercambios de voces y subjetividades. Los enunciados están llenos de matices dialógicos, dado que nuestro pensamiento se origina y se forma en el proceso de interacción con pensamientos ajenos. Las relaciones dialógicas se presentan tanto al estar de acuerdo como en desacuerdo, y permiten cambios y enriquecimiento mutuo en la comprensión. 
De este modo, en la escritura colaborativa emergen procesos dialógicos que acompañan la experiencia de escribir. La dialogicidad en la escritura colaborativa se entiende como el encuentro de voces, en el que los significados son negociados y coconstruidos en un tejido de tensiones, disensos y acuerdos en el proceso de escritura como práctica social. Esto resalta que tiene una intención, hace parte de una comunidad discursiva y se concreta en géneros discursivos que tienen una historicidad.

A su vez, al abordar la escritura colaborativa es necesario interrogarse por el concepto de colaboración. Para iniciar, algunos autores diferencian colaboración de cooperación. Al respecto, Roselli (2016) define que la cooperación se caracteriza por una división de funciones basada en la repartición de la tarea en unidades, para luego ensamblar las partes. Por su parte, la colaboración la explica el proceso colectivo de principio a fin, en el que todos los participantes asumen una postura activa en la realización de una tarea, con una diferenciación horizontal de roles. El autor afirma que en la colaboración no se trata de la aplicación circunstancial de técnicas grupales, sino de promover el intercambio, el compromiso y la participación en la construcción de una cognición compartida.

Por su parte, Duffy (2014) resalta la colaboración como un acto discursivo, una interacción progresiva con los objetos del discurso en una actividad mutua. A pesar de las diferencias establecidas, Storch (2019), en un artículo de revisión sobre escritura colaborativa, plantea que la distinción entre colaboración y cooperación puede ser poco clara, dado que los dos términos han sido utilizados por los investigadores indistintamente.

\section{Metodología}

La revisión siguió los siguientes criterios de inclusión de los estudios: 1) el abordaje de la escritura colaborativa; 2) la fecha de publicación comprendida entre el año 2009 y 2020; 3) los artículos publicados 
en revistas indexadas en idioma español, inglés y portugués; y 4) el tipo de artículo: investigación, revisión y teórico.

La revisión bibliográfica se construyó a partir de la búsqueda de artículos teóricos, de revisión e investigación en las bases de datos: Scopus, ProQuest, Taylor \& Francis, Web of Science, JSTOR y Ebsco. Las palabras clave que se establecieron en la búsqueda fueron: escritura colaborativa, proceso de escritura, producción textual y escritura con tecnología digital. Los 51 artículos que componen el cuerpo de la revisión bibliográfica presentan las siguientes características:

Tabla 1. Presentación de artículos sobre escritura colaborativa.

\begin{tabular}{|c|c|c|c|}
\hline Tipo de artículo & Revista & $\begin{array}{l}\text { Autores y año de } \\
\text { publicación }\end{array}$ & $\begin{array}{l}\text { Temática central del } \\
\text { artículo }\end{array}$ \\
\hline \multirow{11}{*}{$\begin{array}{l}\text { Artículo de } \\
\text { investigación }\end{array}$} & $\begin{array}{c}\text { Australian Journal of } \\
\text { Language and Literacy } \\
\text { Australian Journal of } \\
\text { Language and Literacy }\end{array}$ & $\begin{array}{l}\text { Rojas-Drummond et ál. } \\
\qquad(2020) \\
\text { Stagg y Rajendram (2019) }\end{array}$ & \multirow{11}{*}{$\begin{array}{l}\text { La producción } \\
\text { textual colaborativa } \\
\text { en ambientes } \\
\text { presenciales }\end{array}$} \\
\hline & Infancia y Aprendizaje & $\begin{array}{c}\text { Montanero y Madeira } \\
(2019)\end{array}$ & \\
\hline & Fórum linguistic, Florianópolis & Oliveira (2017) & \\
\hline & Lenguaje & Arenas Hernández (2017) & \\
\hline & Forma y Función & $\begin{array}{c}\text { Vergara Novoa y Perdomo } \\
\text { Cerquera (2016) }\end{array}$ & \\
\hline & $\begin{array}{l}\text { Canadian Journal of } \\
\text { Education }\end{array}$ & Bandenhors et ál. (2016) & \\
\hline & $\begin{array}{c}\text { Research in the Teaching of } \\
\text { English }\end{array}$ & Thompson (2013) & \\
\hline & $\begin{array}{l}\text { Research in the Teaching of } \\
\text { English }\end{array}$ & Godbee (2012) & \\
\hline & Language Arts & Mazer y Potter (2011) & \\
\hline & $\begin{array}{l}\text { Revista española de } \\
\text { pedagogía }\end{array}$ & Castelló et ál. (2010) & \\
\hline & Die Unterrichtspraxi & Roody (2009) & \\
\hline \multirow{3}{*}{$\begin{array}{l}\text { Artículo de } \\
\text { investigación }\end{array}$} & $\begin{array}{l}\text { Australian Journal of } \\
\text { Language and Literacy }\end{array}$ & Adlington y Feez (2019) & \multirow{3}{*}{$\begin{array}{c}\text { La mediación de la } \\
\text { tecnología digital } \\
\text { en la escritura } \\
\text { colaborativa }\end{array}$} \\
\hline & $\begin{array}{c}\text { Assessment \& Evaluation in } \\
\text { Higher Education }\end{array}$ & Guasch et ál. (2018) & \\
\hline & $\begin{array}{l}\text { Innovations in Education and } \\
\text { Teaching International }\end{array}$ & Cho y $\operatorname{Lim}(2017)$ & \\
\hline
\end{tabular}




\begin{tabular}{|c|c|c|c|}
\hline Tipo de articulo & Revista & $\begin{array}{l}\text { Autores y año de } \\
\text { publicación }\end{array}$ & $\begin{array}{l}\text { Temática central del } \\
\text { artículo }\end{array}$ \\
\hline \multirow{18}{*}{$\begin{array}{l}\text { Artículo de } \\
\text { investigación }\end{array}$} & Revista intersaberes & $\begin{array}{c}\text { Araujo Moraes y } \\
\text { Medianeira Tomazetti } \\
(2017)\end{array}$ & \multirow{18}{*}{$\begin{array}{c}\text { La mediación de la } \\
\text { tecnología digital } \\
\text { en la escritura } \\
\text { colaborativa }\end{array}$} \\
\hline & $\begin{array}{l}\text { Revista Lasallista de } \\
\text { investigación }\end{array}$ & Uribe Zapata et ál. (2017) & \\
\hline & Online Information Review & Du et ál. (2016) & \\
\hline & University of Michigan Press & Dougerthy (2015) & \\
\hline & University Michigan press & Odonell (2015) & \\
\hline & Linguagem \& Ensino & Araujo y Hissa (2014) & \\
\hline & $\begin{array}{c}\text { Journal of Adolescent \& Adult } \\
\text { Literacy }\end{array}$ & Yim et ál. (2014) & \\
\hline & Lámpsakos & Collazos et ál. (2014) & \\
\hline & $\begin{array}{l}\text { Educational Technology } \\
\text { Research and Development }\end{array}$ & Woo et ál. (2013) & \\
\hline & University Michigan press & $\begin{array}{l}\text { Melmhan Petezela y } \\
\text { Maneki (2013) }\end{array}$ & \\
\hline & Knowledge Society Journal & Álvarez y Basa (2013) & \\
\hline & The English Journal & Bush y Zuidema (2013) & \\
\hline & Cultura y Educación & Mauri et ál. (2014) & \\
\hline & TESOL Quarterly & Warshauer et ál. (2013) & \\
\hline & Informatic in education & Hadjerrouit (2012) & \\
\hline & $\begin{array}{l}\text { Revista española de } \\
\text { psicología }\end{array}$ & Tapia Ladino et ál. (2012) & \\
\hline & Language and Education & Pirfarré y Fisher (2011) & \\
\hline & Infancia y Aprendizaje & Mauri et ál. (2011) & \\
\hline
\end{tabular}




\begin{tabular}{|c|c|c|c|}
\hline Tipo de artículo & Revista & $\begin{array}{l}\text { Autores y año de } \\
\text { publicación }\end{array}$ & $\begin{array}{l}\text { Temática central del } \\
\text { articulo }\end{array}$ \\
\hline \multirow{6}{*}{$\begin{array}{l}\text { Artículo de } \\
\text { investigación }\end{array}$} & The English Journal & Ridh y Caton (2011) & \multirow{6}{*}{$\begin{array}{c}\text { La mediación de la } \\
\text { tecnología digital } \\
\text { en la escritura } \\
\text { colaborativa }\end{array}$} \\
\hline & Art Education & Yang et ál. (2011) & \\
\hline & Middle School Journal & Kissel et ál. (2010) & \\
\hline & The English Journal & Leigh Tharp (2010) & \\
\hline & Die Unterrichtspraxis & Lornsen (2010) & \\
\hline & $\begin{array}{l}\text { College Composition and } \\
\text { Communication }\end{array}$ & Alexander (2009) & \\
\hline \multirow{7}{*}{$\begin{array}{l}\text { Artículo de } \\
\text { investigación }\end{array}$} & $\begin{array}{c}\text { Revista actualidades } \\
\text { investigativas en educación }\end{array}$ & Gutiérrez (2017) & \multirow{7}{*}{$\begin{array}{c}\text { Escritura } \\
\text { colaborativa } \\
\text { en estudios } \\
\text { comparativos }\end{array}$} \\
\hline & Alfa, São Paulo & Filipeto Simoes (2015) & \\
\hline & $\begin{array}{c}\text { Revista mexicana de } \\
\text { investigación educativa }\end{array}$ & $\begin{array}{l}\text { Guzmán Tinajero y Rojas- } \\
\text { Drummond (2012) }\end{array}$ & \\
\hline & Psicothema & $\begin{array}{l}\text { Fidalgo Redondo et ál. } \\
\qquad(2011)\end{array}$ & \\
\hline & Calidoscópio & Pinheiro (2011) & \\
\hline & Information Review & Shu y Chuang (2011) & \\
\hline & $\begin{array}{c}\text { Journal of Social Work } \\
\text { Education }\end{array}$ & Allwart (2011) & \\
\hline \multirow{2}{*}{$\begin{array}{l}\text { Artículo de } \\
\text { revisión }\end{array}$} & Language Teaching & Storch (2019) & \multirow{2}{*}{$\begin{array}{c}\text { Escritura } \\
\text { colaborativa }\end{array}$} \\
\hline & $\begin{array}{c}\text { Revista de Educación a } \\
\text { Distancia }\end{array}$ & Montenegro y Pujol (2009) & \\
\hline \multirow{3}{*}{ Artículo teórico } & $\begin{array}{c}\text { Propósitos y } \\
\text { Representaciones }\end{array}$ & Roselli (2016) & \multirow{3}{*}{$\begin{array}{c}\text { Aprendizaje } \\
\text { colaborativo } \\
\text { Escritura } \\
\text { colaborativa }\end{array}$} \\
\hline & Walter de Gruyter & Donahue y Lillis (2014) & \\
\hline & College English & Duffy (2014) & \\
\hline
\end{tabular}

Fuente: elaboración propia. 
La revisión bibliográfica arrojó artículos de investigación en un $90 \%$, artículos de revisión en $4 \%$ y artículos teóricos en $6 \%$. El análisis del foco de la conceptualización y del diseño metodológico de cada uno de los estudios permitió identificar tres tendencias que agrupan la literatura referida sobre escritura colaborativa en relación con los ambientes educativos en los que se estudia: 1) producción textual colaborativa en ambientes educativos presenciales; 2) escritura colaborativa en ambientes educativos con mediación de tecnología digital; y 3) estudios comparativos sobre el proceso de escritura colaborativa en ambientes educativos presenciales y virtuales, y en tareas de escritura grupal e individual. Este análisis se complementa con la identificación de tendencias en la literatura revisada sobre la población objeto de investigación, las tecnologías digitales utilizadas en los diseños metodológicos y los modos de organización textual privilegiados en los estudios. Esto permite precisar el panorama de la literatura académica sobre escritura colaborativa.

\section{Resultados}

En este apartado se presentan las tres tendencias identificadas en la revisión de los artículos, se explicitan los aportes más relevantes de los diferentes estudios, se señalan algunos aspectos teóricos y metodológicos comunes, y se identifican limitaciones en la literatura revisada.

\section{La producción textual colaborativa en ambientes educativos presenciales}

En esta primera tendencia, las investigaciones han tomado como población objeto de estudio a sujetos en diferentes momentos del ciclo vital: niños, adolescentes, jóvenes universitarios, estudiantes de doctorado y adultos académicos. Para empezar, se reconocen investigaciones que focalizan en niños de preescolar, quinto y sexto de primaria en procesos de escritura colaborativa de textos 
narrativos. En los estudios se evidencia un interés por la dialogicidad, la interacción y la construcción de audiencias para los procesos de escritura (Montanero y Madeira, 2019; Stagg Peterson y Rajendram, 2019; Rojas-Drummond et ál., 2020). Estos estudios privilegian el abordaje de la escritura desde actividades que implican la interacción con el otro, dado que proponen la construcción conjunta de textos en grupo y en parejas, los que incluyen, a su vez, elementos dialógicos en el proceso de escritura, en el que los participantes crean un texto a partir del intercambio de opiniones, observaciones, coconstruyendo con un otro.

Los estudios resaltan la importancia de generar procesos de colaboración y la construcción de una audiencia que facilite que la escritura se vivencie como una práctica social, en la que los sujetos participantes escriben con otros y para otros. En este sentido, el estudio de Rojas-Drummond y colaboradores (2020) propone que el intercambio comunicativo entre los profesores y los estudiantes en la escuela básica primaria genera un andamiaje que fortalece las competencias de escritura de los niños, desde las relaciones dialógicas y la solución colaborativa de problemas.

El interés por la escritura colaborativa también contempla la población de adolescentes. Los estudios enfocan sus aportes en evidenciar la forma en que la colaboración favorece los procesos de escritura y el aprendizaje de una segunda lengua (Oliveira, 2017; Roody, 2009; Thompson, 2013). A su vez, los hallazgos de estas investigaciones se constituyen en aportes, al afirmar que el trabajo de escritura en parejas y grupos promueve la producción textual, motiva a los estudiantes a participar y potencializa el pensamiento crítico, dado que implica tomar decisiones sobre un tema en el proceso de interacción grupal. Además, los procesos de colaboración se potencializan cuando las actividades de escritura parten de los intereses y motivaciones de los participantes.

La escritura colaborativa también ha sido objeto central de reflexión en el campo de la investigación con jóvenes universitarios. Los estudios evidencian, en sus hallazgos, que la escritura colaborativa implica actividades de diálogo, concertación y revisión conjunta 
antes, durante y después del proceso de escritura (Arenas, 2017; Vergara Novoa y Perdomo Cerquera, 2016). Un aporte significativo de dichos estudios es centrar el interés en la producción textual argumentativa y definir los modos de interacción y negociación de significados como aspectos constitutivos de la producción textual colaborativa. Entre las limitaciones se evidencia que estos estudios no profundizan en las dinámicas particulares de construcción de los textos argumentativos en los grupos participantes.

Los estudios con población adulta conformada por académicos y estudiantes de doctorado resaltan en sus diseños metodológicos los diálogos abiertos sobre los textos y la experiencia de escritura. Estos estudios no solo se centran en el texto, sino en los procesos de construcción dialógica expresados en el entramado de significados y sentidos que se tejen en la comunicación verbal durante la escritura (Bandenhors et ál., 2016; Castelló et ál., 2010; Godbee, 2012; Mazer y Potter, 2011). Un aporte relevante de estos estudios es abordar la escritura colaborativa como una práctica social que trasciende el texto, para involucrar procesos relacionales y conversacionales.

Una limitación identificada en estos estudios es que dejan de lado el análisis sobre la familiaridad e historicidad de las relaciones entre los sujetos que conforman los grupos de escritura. Estos aspectos se mencionan de forma general, pero no se profundizan ni se reconocen como una cuestión que potencializa la confianza, la fluidez en las retroalimentaciones y, por ende, enriquece la colaboración en el proceso de escritura.

La revisión permite identificar que algunos estudios abordan la escritura colaborativa resaltando en el análisis el intercambio de voces, posturas y lecturas del texto en la presencialidad. Lo anterior, permite evidenciar que, en un proceso de colaboración, los sujetos no solo negocian los contenidos del texto, sino sus posiciones frente al mundo, matizan en el diálogo sus propias ideologías y se configura la intersubjetividad en el acto creativo de la escritura (Castelló et ál., 2010; Godbee, 2012; Mazer y Potter, 2011). 
La escritura colaborativa en ambientes educativos con mediación de tecnología digital

La segunda tendencia encontrada en la revisión son los estudios que han abordado la escritura colaborativa con mediación de tecnologías digitales. En estos se identifican tres énfasis: 1) la relación entre los coautores del texto; 2) la comunicación en la escritura colaborativa; y 3) la retroalimentación durante el proceso de producción textual colaborativa.

En el primer énfasis se reconocen estudios que destacan los cambios en la relación entre los autores del texto durante el proceso de escritura colaborativa. Por ejemplo, algunas investigaciones con niños escolarizados y adolescentes identificaron que los blogs, Google Docs, WordPress y las wiki proporcionan — desde la mediación de tecnologías digitales- un aporte al desarrollo del trabajo colaborativo y la interacción en la que se transforman las relaciones de poder entre autores, coautores y lectores, puesto que la tecnología digital puede crear un ambiente de relaciones horizontales, en el que cada sujeto es autor, revisor y lector (Adlington y Feez, 2019; Dougerthy 2015; Hadjerrouit, 2012; Montenegro y Pujol, 2009; Pifarré y Fisher, 2011; Warschauer, Zeng y Park, 2013).

Los autores señalan que el uso de la tecnología digital pone de manifiesto que la interacción e interdependencia en la creación colectiva, para dar lugar a la colaboración, necesita de diferentes elementos de soporte y mediación, que pueden ser brindados por las herramientas digitales. Estos estudios permiten afirmar que la escritura colaborativa con mediación de tecnología digital trasciende el texto y abarca la transformación en las relaciones establecidas entre los actores de la interacción.

Otros estudios que usaron como mediación Facebook, Wiki y Google Docs identificaron que la participación de los estudiantes para el trabajo colaborativo no ocurre automáticamente en la tecnología digital. Se trata de un proceso que se consolida de manera paulatina, en la medida que los colaboradores interactúan entre ellos, a partir de la regulación compartida, que incluye el 
establecimiento de objetivos, el monitoreo y la evaluación grupal de los productos construidos (Araujo Moraes y Medianeira Tomazetti, 2017; Bush y Zuidema, 2013; Cho y Lim, 2017; Rish y Caton, 2011). Por lo tanto, los entornos colaborativos son aquellos que tienen el potencial de promover el aprendizaje para que el estudiante esté en la posición de agente principal de la construcción de su conocimiento.

Así mismo, algunos estudios plantean que los ambientes lúdicos de escritura y la familiarización con el uso de la tecnología digital promueven la comunicación entre los coautores y afianzan los procesos de colaboración. Esta conclusión fue inferida de estudios con jóvenes universitarios, quienes utilizaron salas de chat, foros, juegos de computadora y videos para proponer actividades de escritura que fomenten la colaboración, la creatividad de los participantes y la conexión con sus intereses (Alexander, 2009; Lornsen, 2010).

En estos estudios la escritura colaborativa es asumida como una actividad social que transforma el texto y las relaciones de los coautores, lo que deriva en beneficios para el proceso de aprendizaje (Hadjerrouit, 2012). La escritura colaborativa fomenta el pensamiento crítico, ayuda a aclarar, explicar y reflexionar sobre ideas, permite el intercambio de información y desarrolla habilidades de discusión y comunicación.

En el segundo énfasis se ubican los estudios que resaltan en sus hallazgos las formas de comunicación en la escritura colaborativa con mediación de tecnologías digitales. Algunas investigaciones con estudiantes universitarios resaltan que la colaboración en los procesos de escritura se solidifica por la construcción de un ambiente comunicativo flexible, que se apoya en las amplias posibilidades de la tecnología digital (Cho y Lim, 2017; Mauri et ál., 2011). Los investigadores concluyen que los entornos online se distinguen por las siguientes condiciones comunicativas, que le son propias y que solo se dan juntas en este tipo de entornos: la comunicación en grupo, la comunicación de manera independiente del lugar y del momento, y la comunicación enriquecida por varios recursos 
textuales de la red y mediada por tecnología. Estas condiciones comunicativas son importantes para la escritura colaborativa en la medida que posibilitan la construcción de la intersubjetividad mediada por las posibilidades de las tecnologías digitales.

Así mismo, algunas investigaciones con estudiantes universitarios de nivel de pregrado y posgrado concluyeron que la comunicación constante permitió que sujetos con diferentes enfoques académicos y en lugares distantes, trabajaran juntos. Por consiguiente, se reduce el aislamiento en la escritura académica y se fomenta la colaboración (Du et ál., 2016; Kissel et ál., 2010; Mauri et ál., 2014; Melmhan Petrzela y Manekin, 2013; Yim et ál., 2014).

De esta manera, los estudios evidencian implicaciones prácticas sobre cómo la tecnología digital debe implementarse de manera diferencial para los estudiantes - teniendo en cuenta los niveles de escolaridad - para de esta manera facilitar la construcción colaborativa del conocimiento. Por ejemplo, los estudios enfatizan que, aunque los niños, adolescentes y jóvenes están familiarizados con el manejo de las plataformas virtuales y con la tecnología digital, se requiere un proceso pedagógico que acompañe el uso de estas mediaciones digitales y concertar grupos de escritores con niveles escolares próximos para facilitar la colaboración en la producción textual.

En el tercer énfasis se sitúan los estudios que resaltan el lugar central de la retroalimentación en la escritura colaborativa con mediación de tecnologías digitales. Se comprende por retroalimentación los comentarios, reflexiones y observaciones que los escritores realizan a sus textos en un proceso de coautoría. Por ejemplo, en los estudios con niños de quinto grado de primaria, adolescentes y jóvenes universitarios que utilizan las wikis como mediación, los investigadores identifican que la facilidad proporcionada por la wiki para el trabajo colaborativo está dada por la posibilidad de recibir y ofrecer retroalimentación inmediata.

Los estudios concluyen que la escritura colaborativa implica una mayor responsabilidad frente al documento de trabajo, motiva 
a los estudiantes a participar de manera activa en todos los procesos de escritura y estimula el pensamiento reflexivo (Álvarez y Basa, 2012; Hadjerrouit, 2012; Leigh Tharp, 2010; Tapia Landino et ál., 2012; Uribe Zapata et ál., 2017; Yang et ál., 2011). Los hallazgos de estos estudios reportaron que la escritura colaborativa de los estudiantes en la wiki los involucró en una actividad de aprendizaje colaborativo, partiendo de los comentarios y retroalimentaciones, desde una perspectiva crítica y de apertura a la libre participación, lo que facilitó la coautoría de los textos.

Hallazgos en este mismo sentido fueron identificados por investigadores que analizaron la forma como los aportes y comentarios de los escritores eran utilizados para enriquecer los textos argumentativos de tema libre. Al respecto, concluyeron que la retroalimentación es un proceso central en la escritura colaborativa al evidenciar el papel activo de los coautores (Araujo y Hissa, 2014; Collazos et ál., 2014; Guasch et ál., 2019; Mauri et ál., 2011; Odonell, 2015; Woo et ál., 2013).

Finalmente, se reconoce que los tipos de tecnologías digitales privilegiadas como mediadoras en la escritura colaborativa son las wikis, los foros, las aulas virtuales, la plataforma Moodle, WordPress, Google Docs y los blogs. Algunas investigaciones resaltan las facilidades y ventajas de esta mediación para consolidar la colaboración en el proceso de escritura y la transformación de las relaciones entre autores, coautores y lectores, así como las formas de interacción que se configuran (Adlington y Feez, 2019; Dougerthy, 2015; Pifarré y Fisher, 2011).

A su vez, entre las limitaciones se identifican que los estudios no profundizan en los análisis del texto compuesto, en su coherencia, en su contenido, en el tipo de temas propuestos y en la calidad textual. En los diseños metodológicos de las actividades de escritura mediados por tecnologías digitales se consideran la frecuencia de la participación y el tiempo de uso de la tecnología digital; sin embargo, es relevante preguntarse por el texto que se produce en estos ambientes digitales. 
El aporte identificado en los estudios sobre la evidencia de la incidencia de la retroalimentación en la producción textual, está en consonancia con la dimensión dialógica de la escritura colaborativa y la forma como las tecnologías digitales movilizan a los sujetos en el desarrollo de la escritura colaborativa. La colaboración en la producción de un texto escrito mediado por tecnología digital puede facilitar a los niños de educación básica primaria, adolescentes y jóvenes universitarios asumir distintas responsabilidades en función de una sola meta. El recurso tecnológico sirve de mediador en la interacción y reflexión sobre las ideas que surgen durante el proceso de construcción del texto (Guasch et ál., 2019; Mauri et ál., 2011; Tapia Landino et ál., 2012; Uribe Zapata et ál., 2017; Woo et ál., 2013).

\section{El proceso de escritura colaborativa en estudios comparativos}

Una tercera tendencia agrupa los estudios que analizaron la producción textual a partir de comparaciones entre actividades de escritura con población adolescente y jóvenes universitarios en ambientes educativos presenciales y virtuales, y entre escritura grupal e individual. Se identifican estudios que desarrollan un análisis comparativo entre la producción textual individual asumida como grupo control, y la producción textual en un ambiente grupal y colaborativo. Los hallazgos de las investigaciones evidencian que los grupos que escriben de manera colaborativa se caracterizan por reflexionar y negociar los diferentes componentes textuales - procesos propiciados por la interacción en el trabajo grupal-, mientras que en la escritura individual los escritores solo hacen énfasis en aspectos sintácticos de la composición textual, aspectos relacionados con la ortografía y la estructura formal del texto (Fidalgo Redondo et ál., 2011; Filipeto Simoes, 2015; Gutiérrez, 2016; Guzmán Tinajero y Rojas-Drummond, 2012).

Otros estudios se centran en la comparación entre la virtualidad y la presencialidad en la producción textual. Dicha comparación permite identificar que la presencialidad puede tener un menor 
nivel de incidencia en el trabajo colaborativo, dado que el intercambio comunicativo emerge solo en la interacción presencial. En contraste, en la virtualidad la creatividad y la innovación pueden ser promovidas y facilitadas por la mediación de la tecnología digital; por ejemplo, el registro de la retroalimentación en las plataformas virtuales permite que los participantes retomen en distintos momentos estos aportes en la coconstrucción del texto (Allwart, 2011; Pinheiro, 2011; Shu y Chuang, 2010).

Por su parte, se identifica como limitación de los estudios que, aunque mencionan la relación entre la consolidación de la colaboración, la argumentación y la composición textual, no se profundiza de qué manera la colaboración incide de forma positiva en el nivel de argumentación y la calidad textual, aspecto que puede ser un tema por abordar en futuras investigaciones.

\section{Discusión}

La revisión de los distintos estudios permite reflexionar sobre los aportes que la escritura colaborativa realiza a la concepción de sujeto en el campo educativo, puesto que se avanza en la comprensión de un sujeto que se construye en diálogo con el otro, y que en esta dialogicidad despliega su potencial creativo. Las tendencias de los estudios evidencian que la escritura colaborativa promueve la construcción de conocimiento, en la medida que visibiliza los contrastes y las contradicciones propias de un proceso de construcción conjunta. Las tensiones que emergen en relación con las motivaciones y las singularidades de los sujetos en este proceso constituyen un ambiente propicio para el surgimiento de la novedad.

De esta manera, la escritura colaborativa, tanto en ambientes educativos presenciales como mediados por tecnologías digitales, potencializa el encuentro con el otro. Este involucra la dimensión afectiva y las valoraciones frente al mundo, las intenciones y las motivaciones particulares de los escritores, quienes exponen 
sus posiciones, al tiempo que estas pueden ser movilizadas y transformadas en la producción textual.

La revisión permitió identificar que la escritura colaborativa se estudia a lo largo del ciclo vital; sin embargo, se pueden identificar tendencias en la población que predomina en las investigaciones. La figura 1 muestra que los estudios privilegiaron el análisis de la colaboración en la producción textual de niños (37\%) y universitarios (29\%), principalmente en los estudios con mediación de tecnología digital. En gran medida esto se debe al interés de los estudios por aportar al uso de la tecnología digital en el ámbito educativo. A su vez, esta tendencia evidencia el desafío de la educación actual de fortalecer el diálogo entre la innovación y las tecnologías digitales en los procesos de escritura.

Figura 1. Población objeto de estudio en los estudios sobre escritura colaborativa.

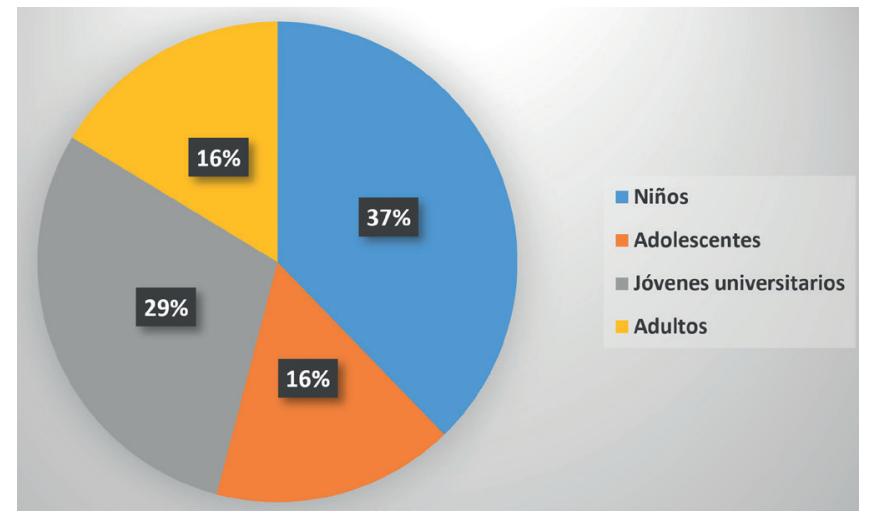

Fuente: elaboración propia.

Por su parte, los estudios que exploraron la escritura colaborativa desde la mediación de tecnologías digitales propusieron en sus diseños: wikis, foros, aulas virtuales, plataforma Moodle, wordpress, Google Docs y blogs. Los análisis resaltan las facilidades y ventajas de estas mediaciones para consolidar la colaboración en el proceso de escritura, a partir de la interacción y el cambio en las relaciones entre los escritores.

La figura 2 ilustra la tendencia de los estudios de acuerdo con el uso de la tecnología digital, la mediación de las wikis (47\%) predomina 
en las investigaciones. Los estudios identifican que la wiki es una herramienta facilitadora del trabajo colaborativo, en la medida que es de fácil uso, permite historial de cambios y la comparación de versiones anteriores del texto, facilita la comunicación asincrónica, promueve la colaboración dado que cada participante se diferencia con un usuario y esto facilita la coconstrucción del texto.

Figura 2. Tecnologías digitales reportadas en los estudios sobre escritura colaborativa.

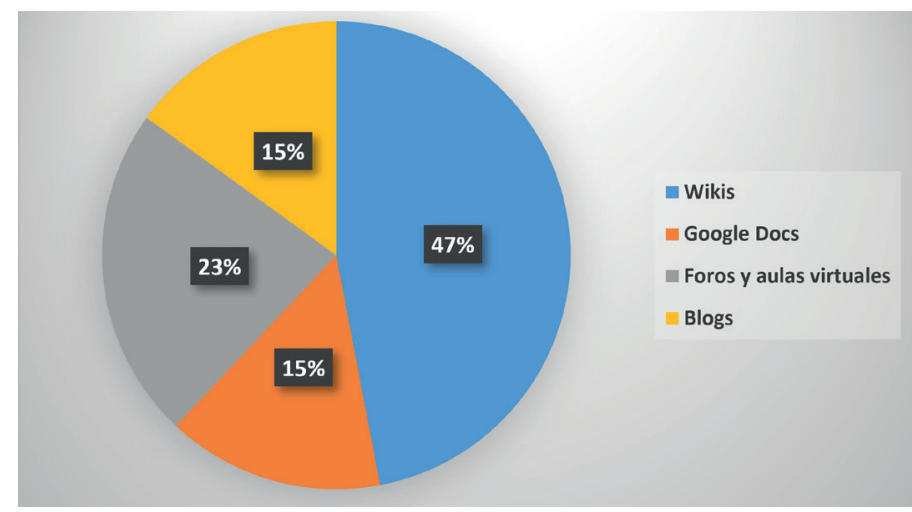

Fuente: elaboración propia.

A su vez, se identificó el predominio de algunos estudios por los modos de organización textual propuestos en relación con la población participante y el ambiente presencial o mediado por tecnología digital. A continuación, en la figura 3 se ilustra esta tendencia.

Figura 3. Modos de organización textual en los estudios sobre escritura colaborativa.

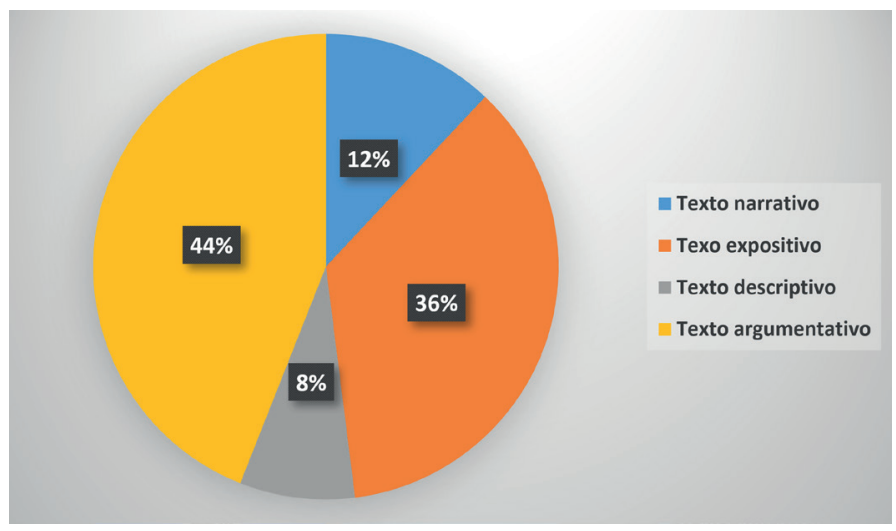

Fuente: elaboración propia. 
En la revisión se pudo identificar una mayor tendencia en la propuesta de escritura colaborativa de textos argumentativos (44\%), en gran parte mediados por tecnologías digitales (wikis, aulas virtuales y foros). Lo anterior pudo responder al interés de los investigadores por explorar el potencial de las tecnologías digitales en la retroalimentación de los escritores y en el seguimiento al cambio de posiciones en la producción textual. Por su parte, los textos expositivos (36\%) y narrativos ( $8 \%$ ) tuvieron mayor presencia en los estudios que realizaron análisis comparativos y en las investigaciones que se enfocaron en ambientes educativos presenciales.

Por otro lado, la revisión bibliográfica permitió identificar limitaciones tales como la poca profundidad y análisis de algunos estudios en aspectos como la historicidad y la familiaridad en las relaciones de los sujetos participantes en la escritura colaborativa, así como en la relación entre colaboración, calidad textual y procesos argumentativos.

\section{Conclusiones}

El estudio de la escritura colaborativa plantea la pregunta sobre cómo aprovechar las herramientas digitales en las prácticas educativas en consonancia con los momentos del desarrollo de los sujetos, los propósitos de la escritura, los géneros discursivos producidos, el contexto sociocultural y las comunidades discursivas para las que se elaboran los textos. Se reconoce que la escritura tiene un lugar fundamental en el aprendizaje de las distintas áreas de conocimiento; en este sentido, es importante articular el trabajo con estudios en el campo de las TIC y los dispositivos móviles en la educación, que analizan el aprendizaje autónomo y colaborativo en diversas áreas (Acevedo-Zapata et ál., 2020; Aparicio-Gómez, 2019; Pedraza-Goyeneche et ál., 2020).

El estudio de la escritura colaborativa aporta a la comprensión del sujeto y de la escritura, y permite entender que el escritor no se define por sí solo y de forma individual, en contraste, se construye 
en la regulación compartida, en la negociación y en el reconocimiento de la alteridad. De esta manera, estudiar la escritura colaborativa conlleva a considerar en los análisis los aspectos relacionales entre los escritores, el texto escrito y su coherencia, el propósito del escrito y la audiencia, y la conexión de los contenidos con la realidad social.

Algunas limitaciones identificadas en la revisión de los estudios son la poca exploración y análisis de aspectos como la historicidad y la familiaridad en las relaciones de los sujetos participantes en la escritura colaborativa, así como el análisis de la relación entre colaboración, calidad textual y procesos argumentativos. Identificar estas limitaciones ofrece una oportunidad para que futuras investigaciones aporten a su comprensión, lo que a su vez permite proponer prácticas educativas que reconozcan que la producción textual colaborativa implica procesos cognitivos, sociales, afectivos y discursivos.

Los estudios evidencian que la escritura colaborativa no es un proceso que se facilita de manera automática por las herramientas digitales. La colaboración exige reconocer la importancia de la alteridad en la constitución de las interacciones y el establecimiento de relaciones de confianza en los grupos de escritura. Esto implica el diseño y acompañamiento de situaciones educativas que convoquen a la participación y al encuentro con el otro, desde una perspectiva de compromiso y coautoría. De esta manera, se interroga el predominio que ha tenido en la escolaridad el trabajo individual y la competencia entre los estudiantes. La revisión invita a privilegiar actividades diseñadas para promover el trabajo colaborativo, y considerar una concepción de sujeto en continua construcción con un otro, el reconocimiento de la dialogicidad como parte constitutiva de la generación de conocimiento y creatividad.

Finalmente, la situación actual, causada por la pandemia de la COVID-19, plantea el desafío en la educación de incorporar y usar las tecnologías digitales, que aparecen como una vía privilegiada para darle continuidad a los procesos educativos en los diferentes niveles de formación. Se trata de un momento de cambio cultural, 
que exige avanzar en las propuestas que favorecen el trabajo colaborativo en la educación en ambientes digitales, para promover el aprendizaje, la construcción de conocimiento, el encuentro con el otro, la corresponsabilidad social y el acompañamiento en el interior de las comunidades.

Al mismo tiempo, la apertura de los niños y de los jóvenes hacia la interacción cotidiana con dispositivos, plataformas y diversidad de tecnologías digitales exige a los investigadores y educadores considerar los aspectos éticos en el uso de estas herramientas en la educación. La utilización ética de las tecnologías digitales implica evitar un uso desmesurado, que puede llegar a afectar negativamente a las personas de manera inmediata o en el futuro (Olcott et ál., 2015). De igual manera, exige reconocer la propiedad intelectual de los textos, proteger la identidad y los datos personales, así como fortalecer desde las prácticas educativas su uso crítico y razonable.

\section{Referencias}

Acevedo-Zapata, S., Pinto-Parra, D. M. y Lemos-Rozo, A. Y. (2020). Mediación pedagógica en la narrativa visual de cursos virtuales introductorios a licenciaturas. Revista Interamericana de Investigación, Educación y Pedagogía (RIIEP), 13 (1) 113-136. https://bit.ly/2PaKyRP

Adlington, R. y Feez, S. (2019). Reading, writing and co-authorship in blogs. Australian Journal of Language and Literacy, 42(1), 19 -29. https:// bit.ly/3gbuzPm

Alexander, J. (2009). Gaming, student literacies and the classroom. College Composition and Communication, 61(1), 35-63. https://www. jstor.org/stable/40593514

Allwart, D. E. (2011). Writing with wikis: a cautionary tale of technology in the classroom. Journal of Social Work Education, 47(3) 597-605 https://doi.org/10.5175/JSWE.2011.20090012 
Álvarez, G. y Basa, L. (2013). ICTS and collaborative learning: a case study of a class blog for improving the writing skills of pre-university students. Universities and Knowledge Society Journal, 10(2), 254-268. https://doi. org/ 10.7238/rusc.v10i2.1740

Aparicio-Gómez, O. Y. (2019). El uso educativo de las TIC. Revista Interamericana de Investigación, Educación y Pedagogía (RIIEP), 12(1), 211-227. https://doi.org/10.15332/s1657-107X.2019.0001.02

Araujo, N. y Hissa, D. (2014). Processo de escrita de uma webaula: proposta de classificação das etapas da produção textual. Linguagem \& Ensino, Pelotas, 17(3),857-877. https://bit.ly/39xW5DX

Araujo Moraes, S. B. y Medianeira Tomazetti, E. (2017). Grupos do facebook e google docs para leitura e escrita na aula de filosofia. Revista Intersaberes, 12(27), 563 - 573. http://dx.doi.org/10.22169/revint. v12i27.1296

Arenas Hernández, K. A. (2017). Características de la regulación socialmente compartida de la escritura: aportes desde una experiencia investigativa. Lenguaje, 45(1), 35-60. https://doi.org/10.25100/lenguaje. v45i1.4613

Bandenhors, C. M., Mcleond, H., Vandering, D., Li, X., Joy, R., Penney, S., Picket, S. y Hesson, J. (2016). The Journey Between There and Here: Stories of a Faculty Writing Group. Canadian Journal of Education, 39(1) 1-26. https://bit.ly/3hH8ubu

Bajtín, M. (1999). Estética de la creación verbal (10. a ed.). Siglo Veintiuno Editores.

Bush, J. y Zuidema, L. (2013). Professional Collaborative Writing: Teaching, Writing, and Learning Together. The English Journal, 102(4), 107-110. https://eric.ed.gov/?id=EJ1017399

Castelló, M., Gonzales, D. y Iniesta, A. (2010). La regulación de la escritura académica en el doctorado: el impacto de la revisión colaborativa en los textos. Revista Española de pedagogía, 1(247), 521-537. https://bit. ly/3hH9SLe 
Collazos, C. A., Ochoa, X., Rodes, V. y Maturana, J. (2014). Creación de Libros Colaborativos en el Marco de la Iniciativa Latin. Lámpsakos, (12), 120-130.https://doi.org/ 10.21501/21454086.1315

Cho, M. H. y Lim, S. (2017). Using regulation activities to improve undergraduate collaborative writing on wikis. Innovations in Education and Teaching International, 54 (1), 53-61. https://doi.org/ 10.1080/ 14703297.2015.1117009

Du, H. S., Chu, S. K., Chan, R. C. y He, W. (2016). Collaborative writing with wikis: an empirical investigation. Information Review, 40(3), 380399. https://doi.org/ 10.1108/OIR-06-2015-0173

Donahue, C. y Lillis, T. (2014). 4 models of writing and text production. En E. Jakobs y D. Perrin (eds.), Handbook of writing and text production (57-78). Walter the Guyter.

Dougherty, J. (2015). Co-Writing, Peer Editing, and Publishing in the Cloud. En T. Dougherty y J. O'Donell, Web Writing (63-72). University of Michigan Press: Digital culture books. https://bit.ly/39Rj0dB

Duffy, W. (2014). Collaboration (in) Theory: Reworking the Social Turn's Conversational Imperative. College English, 76(5), 416-435. https:// www.jstor.org/stable/24238154

Fidalgo Redondo, R., Torrance, M. y Robledo Ramon, P. (2011). Comparación de dos programas de instrucción estratégica y autorregulada para la mejora de la competencia escrita. Psicothema, 23(4), 672-680. https:// bit.ly/30fSOkH

Filipeto Simões, S. C. (2019). Escrita colaborativa e individual em sala de aula: uma análise de textos escritos por alunos do ensino fundamental. Alfa, São Paulo, 63(1), 133-152. https://doi.org/10.1590/1981-57941904-6

Godbee, B. (2012). Toward Explaining the Transformative Power of Talk about, around, and for Writing. Research in the Teaching of English, 47(2), 171-197. https://bit.ly/3gatXcA 
Guasch, T., Espasa, N. y Martínez-Melo, M. (2019). The art of questioning in online learning environments: the potentialities of feedback in writing. Assessment \& Evaluation in Higher Education, 44(1), 111-123. https:// doi.org/ 10.1080/02602938.2018.1479373

Gutiérrez, M. F. (2017). Escritura colaborativa de textos en quinto grado: Razonamiento y argumentación causal sobre un fenómeno físico. Revista actualidades investigativas en educación, 17(1), 1-25. https://doi.org/ 10.15517/aie.v17i1.27291

Guzmán Tinajero, K. y Rojas-Drummond, S. M. (2012). Escritura colaborativa en alumnos de primaria. Revista mexicana de investigación educativa, 17(52), 217-245. http://www.redalyc.org/revista.oa?id=140

Hadjerrouit, S. (2012). Investigating Technical and Pedagogical Usability Issues of Collaborative Learning with Wikis. Informatic in education, 11(1), 45-64. https://bit.ly/304E4tS

Kissel, B., Hataway, J. I. y Wood, K. D. (2010). Digital Collaborative Literacy: Using Wikis to Promote Social Learning and Literacy Development. Middle School Journal, 41(5), 58-64. https://doi.org/10.1080/0094077 1.2010.11461742

Leigh Tharp, T. (2010). Wiki, Wiki, Wiki-WHAT?" Assessing Online CoIlaborative Writing. The English Journal, 99(5) 40-46. https://www.jstor. org/stable/27807190

Lornsen, T. (2010). Online Assignments: Free Web 2.0 Tools in German Language Classes. Teaching German, 43(2), 194-204. https://www.jstor. org/stable/40961808

Lowry, P., Curtis, A. y Lowry, M. (2004). Building a taxonomy and nomenclature of collaborative writing to improve interdisciplinary research and practice. Journal of Business Communication, 41(1), 66-99. https://doi.org/10.1177/0021943603259363

Mauri, T., Clarà, M. y Remesal, M. (2011). La naturaleza del discurso en la escritura colaborativa online: intersubjetividad y elaboración del significado. Infancia y Aprendizaje, 34(2), 219-233. https://doi.org/1010 $.1174 / 021037011795377629$ 
Mauri, T., Colomina, R. y Gispert, I. (2014). Transformando las tareas de escritura colaborativa en oportunidades para aprender: ayuda educativa y uso de rúbricas en la Educación Superior. Cultura y Educación, 26(2), 298-348. https://doi.org/1010.1080/11356405.2014.935111

Mazer, A. y Potter, E. (2011). Profiles and Perspectives: Spilling Ink: Writing in the Play Zone. Language Arts, 88(5), 381-385. https://www. jstor.org/stable/41804292

Melmhan Petrzela, N. y Manekin, S. (2013). The Accountability Partnership: Writing and Surviving in the Digital Age. En J. Dougherty y K. Nawrotzki (eds.), Writing History in the Digital Age (pp. 235-245). University of Michigan Press, Digital culture books. https://doi.org/10.1093/ ahr/119.1.290

Montanero, M. y Madeira, M. L. (2019). Escritura colaborativa encadenada: efectos en la competencia narrativa de estudiantes de Educación Primaria. Infancia y Aprendizaje, 42(4), 915-951. https://doi.org/10.108 0/02103702.2019.1650464

Montenegro, M. y Pujol, J. (2009). Evaluación de la wiki como herramienta de trabajo colaborativo en la docencia universitaria. Revista de Educación a Distancia, (10), 5-15. https://bit.ly/2BAkOLr

Olcott D., Carrera Farran, X., Gallardo Echenique, E. E, y González Martínez, J. (2015). Ética y Educación en la era digital: perspectivas globales y estrategias para la transformación local en Cataluña. RUSC, Universities and Knowledge Society Journal, 12(2), 59-72. http://dx.doi. org/10.7238/rusc.v12i2.2455

O'donell, M. (2015). Science Writing, Wikis, and Collaborative Learning. En J. Dougherty y T. O'Donnell (eds.), Web Writing (47-54). University of Michigan Press, Digital culture books. https://bit.ly/334FEOH

Oliveira, S. B. (2017). Produção escrita colaborativa: o olhar de aprendizes da língua inglesa sobre a arte. Forum Linguistic, 14(2), 21522174. https://doi.org/10.5007/1984-8412.2017v14n2p2152 
Pedraza-Goyeneche, C. E., Amado-Plata, O. F. y Munévar-Garcia, P. A. (2020). Apropiación de dispositivos móviles en educación: una experiencia de sistematización sobre uso pedagógico de TIC en tabletas. Revista Interamericana de Investigación, Educación y Pedagogía (RIIEP), 13(1), 13-30. https://bit.ly/2OZZCBx

Pifarré, M. y Fisher, R. (2011). Breaking up the writing process: how wikis can support understanding the composition and revision strategies of young writers. Language and Education, 25(5), 451-466. https://doi. org/10.1080/09500782.2011.585240

Pinheiro, P. A. (2011). A escrita colaborativa por meio do uso de ferramentas digitais: ressignificando a produção textual no contexto escolar. Calidoscopio, 9(3), 226-239. https://doi.org/10.4013/ cld.2011.93.07

Rish, R. M. y Caton, J. (2011). Building Fantasy Worlds Together with Collaborative Writing: Creative, Social, and Pedagogic Challenges. The English Journal, 100(5), 21-28. https://www.jstor.org/stable/23047798

Rojas-Drummond, S., Barrera, M. J., Hernández, S., Alarcón, M., Hernández, J. y Márquez. A. M. (2020). Exploring the 'black box' What happens in a dialogic classroom? Australian Journal of Language and Literacy, 1(43), 47-67. https://bit.ly/2P1dGL5

Roody, H. L. (2009). Unsere Rockgruppe geht auf Tournee! A Collaborative Writing Project for the Intermediate Level. Teaching German, 42(1), 6873. https://doi.org/10.1111/j.1756-1221.2009.00037.x

Roselli, N. D. (2016). El aprendizaje colaborativo: Bases teóricas y estrategias aplicables en la enseñanza universitaria. Propósitos y Representaciones, 4(1), 219-280. https://doi.org/10.20511/pyr2016.v4n1.90

Shu, W. y Chuang, Y. H. (2010). Wikis as an effective group writing tool: a study in Taiwan Online. Information Review, 36(1), 89-103. https://doi. org/ 10.1108/14684521211206980

Stagg Peterson, S. y Rajendram, S. (2019). Teacher-child and peer talk in collaborative writing. Australian Journal of Language and Literacy, 42(1), 28-31. https://bit.ly/2P3sNDG 
Storch, N. (2019.) Collaborative writing. Language Teaching, 52(1), 4059. https://doi.org//10.1017/S0261444818000320

Tapia Ladino, M., Correa Pérez, R., Ortiz Navarrete, M. y Neira Martínez, A. (2012) Secuencia didáctica para escritura en colaboración en un entorno wiki: propuesta para estudiantes de enseñanza secundaria. Revista española de psicología, 253, 553-577. https://bit.ly/30SrSeX

Thompson, I. (2013). The Mediation of Learning in the Zone of Proximal Development through a Co-constructed Writing Activity. Research in the Teaching of English, 47(3) 247-276. https://bit.ly/305kYDW

Uribe Zapata, A., Ramírez Salazar, D. A. y Henao Alvarez, O. (2017). Exploración de un ejercicio de escritura colaborativa en línea de un grupo de estudiantes de básica primaria. Revista Lasallista de investigación, 14(1), 29-41. https://doi.org/ 10.22507/rli.v14n1a2

Vergara Novoa, A. y Perdomo Cerquera, M. E. (2016). Fortalecimiento de la expresión oral y escrita en inglés a través de un andamiaje de escritura creativa colaborativa: un estudio de diseño desde la cognición distribuida. Forma y Función, 30(1), 117-155. https://doi.org/ 10.15446/ fyf.v30n 1.62417

Warschauer, M., Zeng, B. y Park, Y. (2013). New Ways of Connecting Reading and Writing. TESOL Quarterly, 47(4), 8, 25-830. https://doi. org/:10.1 002/ tesq. 131

Woo, M. M., Chu, S. K. y Li, X. (2013). Peer-feedback and revision process in a wiki mediated collaborative writing education. Educational Technology Research and Development, 61, 279-309. https://doi.org/ 10.1007/s11423-012-9285-y

Yang, G. M., Suchan, T. y Kundu, R. (2011). Collaborative, Interactive, and Dialogic Platform for Teaching and Learning Art Education, 64, 4853. https://doi.org/ 10.1080/00043125.2011.11519136

Yim, S., Wachabers, M., Zheng, B. y Lawrence, J. F. (2014). Cloud-Based Collaborative Writing and the Common Core Standards. Journal of Adolescent y Adult Literacy, 5, 243-25. https://doi.org/10.1002/jaal.3

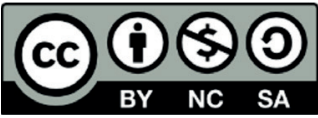

